\title{
Application of new type anti-corrosion material in chemical industry
}

\author{
Juan $\mathrm{Mu}$ \\ Wuxi Institute of Arts and Technology, Wuxi Jiangsu, 214200, China
}

Keywords: New type anti-corrosion material, Metallic material, Application.

\begin{abstract}
With constant development and prosperity of social economy, China's industrial domain has achieved great development, so has the chemical industry. During the specific process of chemical product manufacturing, corrosion of chemical equipment is a common phenomenon. How to effectively prevent corrosion is a significant issue brining trouble to chemistry industry. Based on this, the author makes a brief discussion on the application of new type anti-corrosion material in chemical industry for the purpose of better serving for chemical industry.
\end{abstract}

\section{Introduction}

Corrosion in broad sense refers to chemical or electrochemical reaction between materials with surrounding environment leading to the materials' damage or deterioration, and in narrow sense refers to the process of reaction between metallic materials with physical or chemical substances in the environment leading to the damage to metallic material's structure function. Majority of equipment in chemical industry are corrosive which will bring serious impact to chemical industry.

\section{Causes and types of corrosion}

\section{Cause of corrosion}

The reason why metallic materials are corrosive is determined by its structure and property which is the fundamental reason. Temperature and humidity in exterior environment and other substances exposed to metallic materials are the objective conditions for the corrosion of metallic materials. In industrial domains especially chemical industry, there exist a large amount of medium in chemical enterprise environment such as carbon dioxide, sulfur dioxide, and hydrogen peroxide. Some are toxic and harmful, and some are influenced by high temperature, humidity and other factors in the environment. The comprehensive influence makes the metallic materials inevitable have corrosion once they are fully infused interiorly and exteriorly.

\section{Types of corrosion}

Firstly, analyzing from the appearance of metallic materials, metallic corrosion is divided into local corrosion and general corrosion. Local corrosion mainly include corrosion of small pores, corrosion during the fabrication process and corrosion between metallic gaps. Regarding general corrosion, there will cover a layer of corrosion film in the surface of metal which can effectively reduce the speed and degree of metallic materials' corrosion so as to protect metallic materials. From the reasons of corrosion, it can be divided into physical corrosion, chemical corrosion, and electric corrosion. Physical corrosion mainly refers to that metallic materials corrode through simply physical dissolution in the most basic external environment. Chemical corrosion refer to the reaction between the surface of metallic materials and chemical medium to destroy metallic property. Electric corrosion, as the name suggests, means the reaction between metallic materials and electrolyte, with 
the obvious characteristic that electric current will be generated constantly during the reaction process.

\section{China's current chemical equipment and the anti-corrosion status}

With the advent of the Third Industrial Revolution, global economy has achieved great development and manufacturing industry under this context has obtained obvious progress. But it is known to all that the cost of purchasing mechanic equipment in chemical industry is very high, ten thousand at lowest and several million or billion at most. The costs and expenses of purchasing fixed assets are inevitable during the process of chemical industry development. But due to its own reasons, machinery equipment are basically in the environment where corrosive medium exist. The machinery equipment are objects get rusty gradually, in addition to the physical, chemical or electric reaction to corrosive medium in chemical industry environment, it will greatly accelerate the speed of machinery equipment to get rusty, so that the machinery equipment corrosion degree in chemical industry is higher than other industries. Thus, actively discovering anti-corrosion materials in chemical industry is necessary and feasible, which requires for starting from different procedures trying best to slow down the corrosion speed of machinery equipment. It would lengthen the lifetime of machinery equipment, reduce the cost waste resulted from fast corrosion, but can also realize the double objectives of economic development and natural environment friendly co-existence.

As machinery equipment corrosion in chemical industry are attracting people's attention, regarding the focus issue on how to prevent corrosion, people gradually shift the focus to the application of anti-corrosion materials. This benefits from the widely application of anti-corrosion and corrosion-resistant materials in construction material, machinery pipelines and other installation procedures, making chemical industry have to attach great importance to it. Meanwhile, the specific application of machinery equipment anti-corrosion materials in chemical industry domains and its publicity prospect are promising and expecting in the future.

\section{Corrosion hazard and the significance of anti-corrosion}

Metallic corrosion leads to large amount of metallic resource waste and the scrapping of metallic equipment, bring great hazard to chemical companies' profit making and the whole national economy, even bring about disastrous accident to harm human life and property security. During the process of increasing corrosion, in China's national economy, a great number of metallic materials must be scrapped and the majority of steel material in the world are corroded. Economic damage resulted by corrosion is inestimable and even untreatable. It is the incurable disease in metallic material field. But the corrosion can be alleviated through scientific and reasonable management methods and approaches to reduce damages. It can be seen that anti-corrosion has significant positive function on the sustainable development of national economy and world economy [1].

\section{Analysis on the application of routine anti-corrosion materials in chemical equipment}

\section{Principles to select anti-corrosion materials in chemical equipment}

During specific manufacturing chemical materials, ordinary carbon steel are usually used as it is acquired easily from broad sources with low costs but good mechanical properties. The application of this ordinary chemical material carbon steel can in normal environment will not result in the acceleration of corrosion speed or other side effect. But once applied in environment with high concentration corrosive materials or high temperature or humidity, in addition to their poor anti-corrosion ability, it is easily to get corroded and damaged. So when selecting materials of chemical equipment, the first thing to be considered is the equipment's anti-corrosion ability such as the operating pressure and specific temperature of the machinery equipment, and the concentration and degree of corrosive medium contacting with equipment surface because the high or low temperature, humidity, high pressure and vacuum change in external environment will impact the 
corrosion to equipment. Secondly, during the anti-corrosion selection of chemical equipment, the specific using duration should be considered, the using limit for reaching the basic chemical production must be well known so that the chemical equipment can be scrapped with controllable corrosion speed and degree in the corresponding scope.

\section{Development and application of anti-corrosion materials}

While China's manufacturing industry made great achievements, metallic materials and equipment in chemical industry are getting corrosive faster and faster. General chemical equipment's making material is ordinary carbon steel which has low anti-corrosion ability requiring more and more equipment to upgrade so that costs are increasing. Economic loss with less human factors attracts more and more attention and focus. How to use related technology and anti-corrosion materials to alleviate the corrosion speed of metallic materials and lengthen the lifetime of metallic materials requires for discovering anti-corrosion materials. There are relation and connection between people's discovery and the selection of anti-corrosion materials. Basically, anti-corrosion materials people are seeking can be categorized into high polymer material, organic non-metallic material, and metallic material.

When selecting specific materials, structural materials mainly use steel which has limited anti-corrosion ability however. To gradually prevent chemical equipment from being corroded very fast even in complicated tough environment, the application of alloy and high polymer material have achieved related development. For example, bi-directional stainless steel, low alloy steel, etc. The application technology of anti-corrosive non-metallic materials is mature in foreign countries with relatively high frequency because its anti-corrosion ability can be achieved through gradually intensification with the application depth and width strengthening, showing a trend of replacing nonferrous metals and other anti-corrosion metallic materials. China is strengthening and updating in the application of this domain.

\section{Application of surface coating anti-corrosion materials}

Currently, the application of surface coating anti-corrosion material is the highest among all so that its application is the most widely and common. For effectively reducing the machinery equipment maintenance costs, improving its using efficiency and lifespan, it is necessary to find out strong and powerful surface coating anti-corrosion materials which can be the easiest and most direct way for application. In addition, its application can gradually improve the equipment anti-corrosion ability, which is what other technology can match. Specific application mainly include two types: the first is the application of lining technology, the major structural materials of which are high intensity materials such as glass fiber reinforced plastics with strongly anti-corrosively materials as the lining layer This kind of lining technology is mainly applied to chemical equipment and pipelines. For example, the lining steel pipe in ceramics has good anti-corrosion property and high temperature resistance. So pipeline transport with corrosive medium in chemical industry mainly applies lining technology widely. The second is coating technology with electroplating technology as the major method, which mainly applies electrochemical method to improve the appearance of metallic equipment, and improve its anti-corrosion property and abrasion resistant performance so that it has good application effect in chemical industry [2].

\section{New type anti-corrosion material}

SPUA as a new type anti-corrosion material is produced through constantly exploring based on predecessors' massive theories and practice, improving chemical equipment's anti-corrosion property and protecting environment accordingly. The technology is a green and pollution-free new technology with obvious characteristics compared with traditional anti-corrosion materials. Firstly, SPUA is not sensitive to exterior environment perception due to its non-reactivity and stagnancy, unable to react to temperature and humidity in exterior environment, and free from disturbance form exterior environment, which makes it easily adapt to complicated environment where chemical 
equipment run. Secondly, SPUA contains no artificial catalyst and can produce a rapidly solid-state effect which makes the spraying in any aspects and angel to shape rapidly remaining no other traces. This has reduced the equipment costs greatly with uniform spraying and good effect. In addition SPUA is available for specific operation based on specific requirement in chemical equipment construction such as the area and thickness of the construction object to rapidly reach the construction requirement rapidly and achieve the expected effect through one-time construction. At last, SPUA has strong heat stability making the original shape remain the same within an extremely large temperature range. This has brought great convenience to specific operation of chemical equipment.

\section{Application method of new type anti-corrosion materials}

New type anti-corrosion materials specific application methods are as below. Firstly during removing the rust in chemical equipment, it can help eliminate the dust and previous paint on the surface of chemical equipment and the sharp object left in previous wielding so as to make the surface of the whole chemical equipment clean and smooth without impurities. Secondly, spray paint to chemical equipment with strongly professional technology based on the specific shape, performance and the needed color of the chemical equipment. At last, dry by airing. As SPUA has the property of rapid solidification, after spraying with SPUA technology to chemical equipment, the equipment can be put into normal use after a short period. This can greatly improve the production efficiency of chemical equipment and increase the overall economic profits in the whole chemical industry.

\section{Innovation of SPUA technology}

The specific operation procedure of applying SPUA to chemical equipment not only with for specific external construction operation based on the shape, size, and thickness of chemical equipment but also for internal construction inside chemical equipment in short solidification time for quick utilization. Secondly, SPU has properties of thermostability, humidity resistance, resistance to strong alkali and strong acid and strong anti-corrosion ability to help chemical equipment operate in extremely complicated and tough environment with corrosion speed slowed down accordingly. In addition, the effect after construction is good in a very short time, easy for operation, at low costs and with huge promotion space. At last, SPUA technology is not sensitive to moist and high temperature in the air compared with other technologies so that it is more suitable for application in complicated environment. Almost not influenced by external environment, SPUA technology is more popular in specific application [3].

\section{Conclusion}

To sum up, specific application of new type anti-corrosion materials in chemical industry is the general trend. Its application is not only motivated by the sustainable development in chemical industry but also is a concrete embodiment of eco-environment protection presenting the state of human-nature harmonious co-existence.

\section{Reference}

[1] Yan Yangyang. Studies on the application of modern new type anti-corrosion material in chemical equipment, Non-State Running Science \& Technology Enterprises, 2014, 07:66.

[2] Yan Gaoxiang. Research and manufacturing of new type water based steel anti-corrosion coating material,Yangtze University, 2012.

[3] Wang Jiamin. Application of spray coating polyuria in chemical anti-corrosion field, Process Equipment \& Piping, 2011,02:65-68. 